\title{
Low Complexity Fluctuation Measurement in Image Processing Considering Order
}

\author{
Tareq Khan \\ School of Engineering Technology, Eastern Michigan University, USA
}

\begin{tabular}{|c|c|}
\hline Article Info & ABSTRACT \\
\hline Article history: & \multirow{10}{*}{$\begin{array}{l}\text { The standard deviation can measure the spread out of a set of numbers and } \\
\text { entropy can measure the randomness. However, they do not consider the } \\
\text { order of the numbers. This can lead to misleading results where the order of } \\
\text { the numbers is vital. An image is a set of numbers (i.e. pixel values) that is } \\
\text { sensitive to order. In this paper, a low complexity and efficient method for } \\
\text { measuring the fluctuation is proposed considering the order of the numbers. } \\
\text { The proposed method sums up the changes of consecutive numbers and can } \\
\text { be used in image processing applications. Simulation shows that the } \\
\text { proposed method is } 8 \text { to } 33 \text { times faster than other related works. } \\
\text { Copyright } \odot 2018 \text { Institute of Advanced Engineering and Science. } \\
\text { All rights reserved. }\end{array}$} \\
\hline Received Feb 5, 2018 & \\
\hline Revised Jul 8, 2018 & \\
\hline Accepted Jul 28, 2018 & \\
\hline Keyword: & \\
\hline Entropy & \\
\hline Fluctuation & \\
\hline Image processing & \\
\hline Standard deviation & \\
\hline Variance & \\
\hline \multicolumn{2}{|l|}{ Corresponding Author: } \\
\hline \multicolumn{2}{|c|}{ Tareq Khan, } \\
\hline \multicolumn{2}{|c|}{ School of Engineering Technology, } \\
\hline \multicolumn{2}{|c|}{ Eastern Michigan University, } \\
\hline \multicolumn{2}{|c|}{118 Sill Hall, Ypsilanti, MI 48197, USA. } \\
\hline \multicolumn{2}{|c|}{ Email: tareq.khan@emich.edu } \\
\hline
\end{tabular}

\section{INTRODUCTION}

Standard deviation and variance [1] measure how far a set of numbers are spread out from their average value and they give an indication of the fluctuation or chaotic nature of the numbers. For instance, let's consider an array of numbers, $S_{1}=[2,2,2,2,9,9,9,9]$. The standard deviation of the elements of $S_{1}$ is 3.74 and the variance is 14 . Now, let's reorder the numbers of $S_{1}$ and let's consider $S_{2}=[2,9,2,9,2,9,2,9]$. For $S_{2}$, the standard deviation is 3.74 and the variance is 14 . Here, we see that standard deviation and variance do not change even if the numbers are reordered. The numbers in $S_{2}$ has more changes with respect to its next number than in $S_{1}$, and $S_{2}$ fluctuates more than $S_{1}$. However, standard deviation and variance cannot acknowledge this difference.

Let's consider a 2D case of numbers. An image is a 2D array of numbers i.e. pixel values [2]. Figure 1a and Figure $1 \mathrm{~b}$ shows two artificial images where they have the same number of white and black pixels. In an 8 bit grayscale image, the white pixel has the value of 255 and the black pixel has the value of 0 . Fig. 2a and Fig. $2 \mathrm{~b}$ shows two natural grayscale images having different textures. The standard deviation, variance, and entropy of the image pixels in Figure 1 and in Figure 2 are shown in Table 1 and in Table 2 namely. From Table 1, we see that the standard deviation, variance, and entropy [3]-[5] of Figure 1a and Figure 1b are exactly the same. However, intuitively Figure $1 \mathrm{~b}$ is more random or unpredictable than Figure 1a. From Table 2, we see that the standard deviation, variance, and entropy of Figure $2 \mathrm{a}$ and Figure $2 \mathrm{~b}$ are very close even though they have different textures. For instance, Figure $3 \mathrm{a}$ and Figure $3 \mathrm{~b}$ shows the changes of pixel values with respect to its adjacent left pixel value for row 127 for Figure $2 a$ and Figure $2 b$ namely. Here, we see that Figure $2 \mathrm{~b}$ has more fluctuations of pixel values than Figure $2 \mathrm{a}$. Quantitively, average absolute change for Figure $3 \mathrm{a}$ is 9.34 and Figure $3 \mathrm{~b}$ is 22.49. 
The standard statistical measures such as standard deviation, variance, and entropy cannot recognize the difference between the image pairs in Figure 1 and Figure 2 because they do not consider the order of the numbers. In an image, the order of the numbers (i.e. pixel values) are significant because changing their order will change the image. Thus if these standard statistical methods are used to find the randomness or fluctuation of the texture of an image, they may lead to misleading results. In this paper, an efficient solution of this problem is proposed. The proposed method sums up the changes of consecutive numbers and can recognize the different texture of images even though they have same standard deviation, variance, and entropy. The proposed method can be used to generate a feature vector in classifying textures of stone [6], wood [7], batik motif [8] etc.

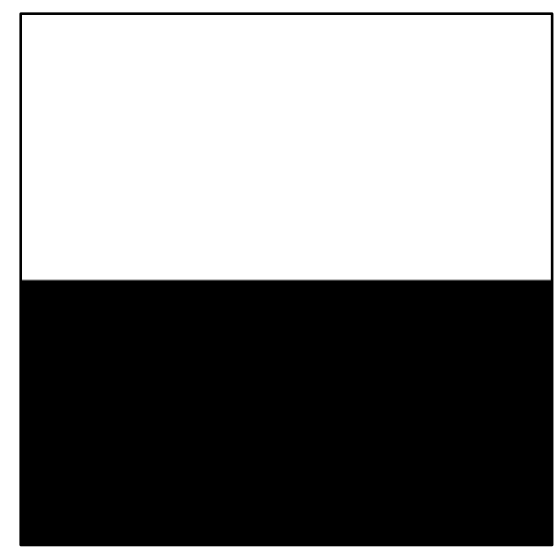

(a)

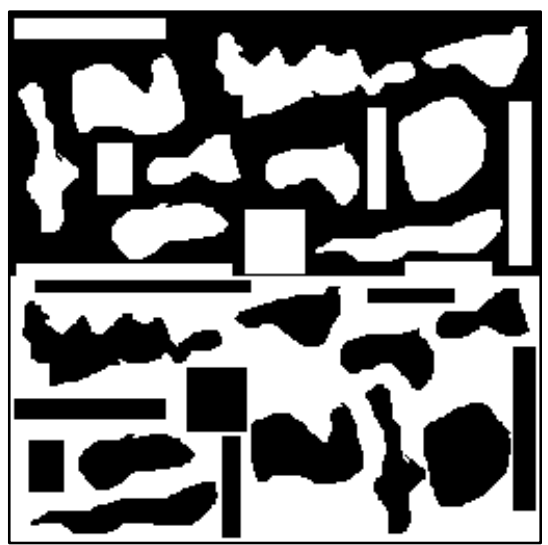

(b)

Figure 1. Artificial images having the same number of white and black pixels. (a) Pixels distributed in an orderly fashion with fewer fluctuations; (b) Pixels distributed randomly having more fluctuations

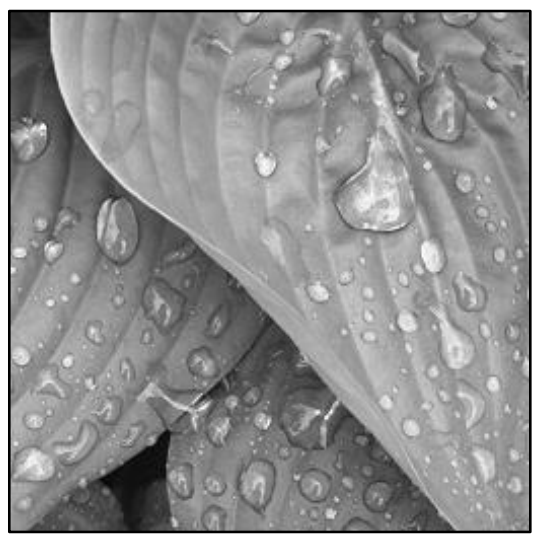

(a)

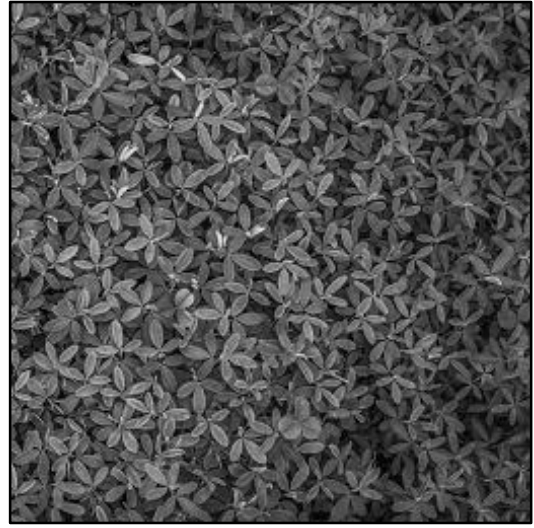

(b)

Figure 2. Natural images having different textures. (a) Pixels distributed having fewer fluctuations; (b) Pixels distributed having more fluctuations

Table 1. Standard Statistical Measurement of Figure 1

\begin{tabular}{cccc}
\hline Figure & Standard Deviation & Variance & Entropy \\
\hline Figure 1a & 127.50 & 16256.25 & 1 \\
Figure 1b & 127.50 & 16256.25 & 1 \\
\hline
\end{tabular}

Table 2. Standard Statistical Measurement of Figure 2

\begin{tabular}{cccc}
\hline Figure & Standard Deviation & Variance & Entropy \\
Figure 2a & 37.7375 & 1424.10 & 7.1794 \\
Figure 2b & 37.7397 & 1424.30 & 7.2472 \\
\hline
\end{tabular}




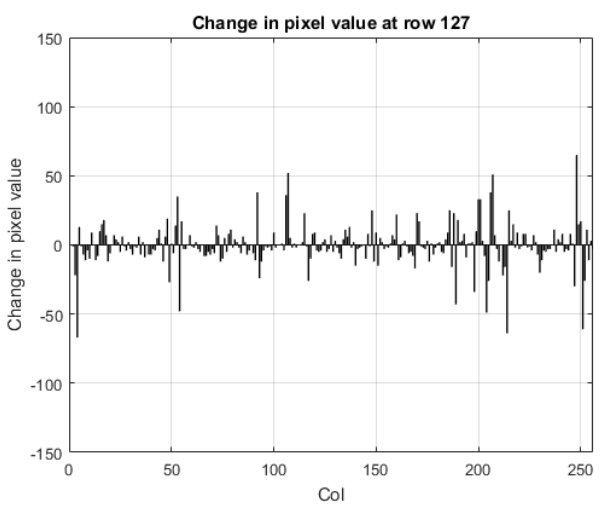

(a)

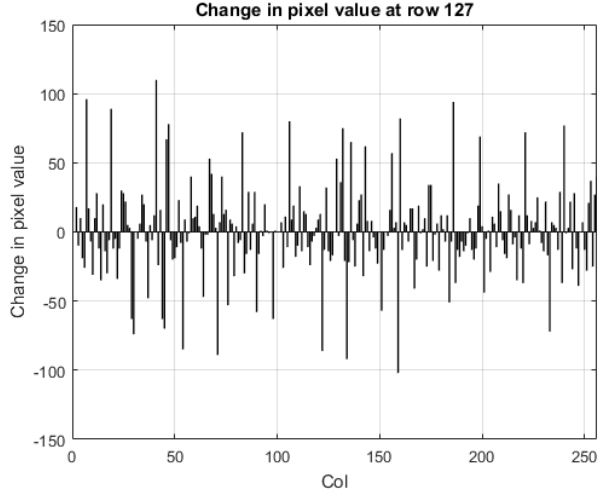

(b)

Figure 3. Changes of the pixel value at row 127 with respect to its left pixel. (a) Changes of pixel values for Figure 2a.; (b) Changes of pixel values for Figure 2b

\section{THE PROPOSED METHOD}

The proposed fluctuation measurement method, $f_{I D}$, for the 1D array is expressed in (1). Here $x_{i}$ is the $\mathrm{i}_{\text {th }}$ element of the array and $N$ is the size of the array. The numerator contains the sum of the squared changes of consecutive numbers and the denominator contains the total number of changes.

$$
f_{1 D}=\frac{\sum_{i=1}^{N-1}\left(x_{i}-x_{i+1}\right)^{2}}{N-1}
$$

For 2D case, such as an image, the proposed fluctuation measurement method, $f_{2 D}$, is expressed in (2), (3), and (4). Here $\left(x_{i}, y_{j}\right)$ is the number at the $\mathrm{i}_{\mathrm{th}}$ column and the $\mathrm{j}_{\mathrm{th}}$ row. $X$ and $Y$ are the total columns and total rows namely. Here in (2), $f_{h}$ represents the fluctuation in the horizontal direction and $f_{v}$ in (3) represents the fluctuation in the vertical direction. Finally, $f_{2 D}$, in (4) is the mean of $f_{h}$ and $f_{v}$ multiplied by a factor $n$. For instance, $n$ could be the maximum pixel value, i.e. 255 for an 8-bit image.

$$
\begin{aligned}
& f_{h}=\frac{\sum_{j=1}^{Y} \sum_{i=1}^{X-1}\left(\left(x_{i}, y_{j}\right)-\left(x_{i+1}, y_{j}\right)\right)^{2}}{(X-1) Y} \\
& f_{v}=\frac{\sum_{i=1}^{X} \sum_{j=1}^{Y-1}\left(\left(x_{i}, y_{j}\right)-\left(x_{i}, y_{j+1}\right)\right)^{2}}{X(Y-1)} \\
& f_{2 D}=\frac{\left(f_{h}+f_{v}\right)}{2} \times n
\end{aligned}
$$

\section{RESULTS}

Using (1), the fluctuation of $S_{1}$ is 7 and the fluctuation of $S_{2}$ is 49 Thus, the proposed method can acknowledge the existence of the frequent changes in $S_{2}$ and give higher fluctuation than $S_{1}$, even though both $S_{1}$ and $S_{2}$ have the same elements. Table 3 and Table 4 show the fluctuation for the image pairs in Figure 1 and Figure 2 using the proposed method namely. Table 3 shows that the fluctuation for Figure $1 \mathrm{~b}$ is much larger than Figure 1a. From Table 4, we see that the fluctuations of Figure $2 b$ is much larger than Figure 2a. Thus, the proposed method can recognize the different amount of fluctuations between the artificial image pairs in Figure 1 and between the natural image pairs in Figure 2. On the other hand - the standard deviation, variance, and entropy are unable to appreciate the different amount of fluctuations 
between the image pairs in Figure 1 as shown in Table 1 and between the image pairs in Figure 2 as shown in Table 2.

Table 3. Fluctuation measurement of Figure 1 using proposed method

\begin{tabular}{cccc}
\hline Figure & Horizontal fluctuation $\left(f_{h}\right)$ & Vertical fluctuation $\left(f_{v}\right)$ & Fluctuation $\left(f_{2 D}\right)$ \\
\hline Figure 1a & 0 & 1 & 127.50 \\
Figure 1b & 4.87 & 5.80 & 1360.33 \\
\hline
\end{tabular}

Table 4. Fluctuation measurement of Figure 2 using proposed method

\begin{tabular}{cccc}
\hline Figure & Horizontal fluctuation $\left(f_{h}\right)$ & Vertical fluctuation $\left(f_{v}\right)$ & Fluctuation $\left(f_{2 D}\right)$ \\
\hline Figure 2a & 41.05 & 33.39 & 9491.24 \\
Figure 2b & 77.68 & 76.89 & 19707.70 \\
\hline
\end{tabular}

One existing method, mentioned in [9]-[11], for finding the fluctuation of an image could be to use a local standard deviation filter. This method returns a 2D array where each output pixel contains the standard deviation of the 3-by-3 neighborhood around the corresponding pixel in the input image. Symmetric padding is used for the pixels in the border. Taking an average of the returned $2 \mathrm{D}$ array can indicate the fluctuation of the image. As this method uses $3 \times 3$ neighboring pixels to calculate the standard deviation of each pixel, the pixel positions and orders are considered in this method. Local entropy filter can also implement in the same way where entropy is calculated instead of standard deviation. In Table 5, the fluctuation is measured in Figure $2 \mathrm{a}$ and Figure $2 \mathrm{~b}$ using local standard deviation filtering, local entropy filtering and using the proposed method. Here, we see that all of these methods correctly reports higher fluctuation in Figure $2 \mathrm{~b}$ than in Figure 2a.

One shortcoming of the local standard deviation filtering and local entropy filtering methods are their time complexity. In these methods, the standard deviation (or entropy) of 9 pixels needs to be calculated for each pixel in the image. However, in the proposed method, only a difference between two pixels needs to be calculated. The local standard deviation filtering, local entropy filtering, and the proposed method were implemented (without implementing vectorization - for proper comparison) in a computer having Intel Pentium CPU $2117 \mathrm{U}$ running at $1.8 \mathrm{GHz}$. The execution times are shown in Table 6. Here, we see that the proposed method is the fastest having the lowest execution time. The proposed method is approximately 8 times faster than local standard deviation filtering and approximately 33 times faster than local entropy filtering method. Fourier spectra methods [2], [12] can be used to measure fluctuation, however, they have higher computational complexity than [9] and the proposed method - and will require more execution time.

Table 5. Fluctuation measurement of Figure 2

\begin{tabular}{cccc}
\hline Figure & Local Standard Deviation Filtering & Local Entropy Filtering & Proposed Method \\
\hline Figure 2a & 10.71 & 2.82 & 9491.24 \\
Figure 2b & 24.58 & 3.03 & 19707.70 \\
\hline
\end{tabular}

Table 6. Comparison of execution time (in Seconds)

\begin{tabular}{cccc}
\hline Figure & Local Standard Deviation Filtering & Local Entropy Filtering & Proposed Method \\
\hline Figure 2a & 3.39 & 13.50 & 0.39 \\
Figure 2b & 3.38 & 13.56 & 0.37 \\
\hline
\end{tabular}

\section{CONCLUSION}

Entropy, standard deviation, and variance may lead to misleading results if they are used to measure randomness or fluctuation. In this paper, a low complexity and fast fluctuation measurement method is proposed considering the order of the numbers for both 1D and 2D cases. The proposed method can be used in image processing applications such as classifying texture.

\section{REFERENCES}

[1] R. Witte and J. Witte, "Statistics," Wiley, 2009.

[2] W. K. Pratt, "Digital Image Processing," Wiley-Interscience, 2007. 
[3] T. Kvalseth, "On the measurement of randomness (uncertainty): a more informative entropy," Entropy, vol/issue: 18(5), pp. 159, 2016.

[4] C. Shannon, "A mathematical theory of communication,” Bell System Technical Journal, vol/issue: 27(4), pp. 623656, 1948.

[5] Y. Wua, et al., "Shannon entropy based randomness measurement and test for image encryption," Information Sciences, Elsevier, vol. 2016, pp. 1-23, 2011.

[6] P. V. Kumar, et al., "Stone Image Classification Based on Overlapped 5-bit T-Patterns Occurrence on 5-by-5 Sub Images," International Journal of Electrical and Computer Engineering (IJECE), vol/issue: 6(3), pp. 1152-1160, 2016.

[7] A. Fahrurozi, et al., "Wood Classification Based on Edge Detections and Texture Features Selection," International Journal of Electrical and Computer Engineering (IJECE), vol/issue: 6(5), pp. 2167-2175, 2016.

[8] I. Nurhaida, et al., "Texture Fusion for Batik Motif Retrieval System," International Journal of Electrical and Computer Engineering (IJECE), vol/issue: 6(6), pp. 3174-3187, 2016.

[9] Standard deviation filters, $2018 . \quad$ http://spatialanalyst.net/ILWIS/htm/ilwisapp/filter_types_standard_deviation_filters.htm.

[10] Local standard deviation of image, 2018. https://www.mathworks.com/help/images/ref/stdfilt.html.

[11] S. S. Singh, et. al, "Local contrast enhancement using local standard deviation," International Journal of Computer Applications (0975 - 888), vol/issue: 47(15), pp. 31-35, 2012.

[12] M. Coggins and A. K. Jain, "A spatial filtering approach to texture analysis," Pattern Recognition Letters, vol/issue: 3(3), pp. 195-203, 1985.

\section{BIOGRAPHIES OF AUTHORS}

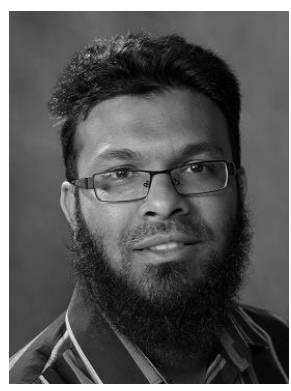

Dr. Tareq Khan received his Ph.D. degree from the department of Electrical and Computer Engineering of University of Saskatchewan, Canada. Dr. Khan is now an Assistant Professor in the School of Engineering Technology of Eastern Michigan University, USA. To date, Dr. Khan has authored (and co-authored) 2 books, 1 book chapter, 18 peer-reviewed journals and 26 international conference papers. He currently has 2 US patents granted. In addition to his academic research, he has also industrial experiences on embedded system projects such as designing pre-paid electricity and gas metering system, automatic meter reading (AMR), data accusation and monitoring systems etc. His research interests include image processing, smart home, embedded systems targeting healthcare applications, Internet of Things (IoT), machine learning, and capsule endoscopy. He is a member of the Institute of Electrical and Electronics Engineers (IEEE). 